\title{
Relay Selection for Flexible Multihop Communication via Competitive Spectrum Leasing
}

\author{
${ }^{1}$ Igor Stanojev and ${ }^{2}$ Aylin Yener \\ ${ }^{1}$ University of Wisconsin \\ Platteville, WI 53818 \\ ${ }^{2}$ The Pennsylvania State University \\ University Park, PA 16802 \\ stanojevi@uwplatt.edu, yener@ee.psu.edu
}

\begin{abstract}
Communication gains from relaying can be acquired even in the absence of altruistic or dedicated relays. To accommodate this, spectrum leasing for cooperation paradigm prescribes that the potential relaying node is rewarded for its relaying role with a fraction of source's bandwidth. Recently, a twohop spectrum leasing scheme was proposed that enables the source to employ the relay only to an extent it finds beneficial, preserving the remaining bandwidth for its direct transmission, if necessary. Such a solution adds flexibility that can be particularly useful for implementation in networks involving more than two hops. In this paper, we extend this setup to include the relay selection problem in a two-hop network scenario with multiple potential relays. Since the potential relays are selfish nodes, their competitive attitude for accessing the spectrum needs to be taken into account. In addition to Stackelberg game framework used in the original setting with one relay, auction theory is applied to model the relay selection process. Analysis and numerical results are provided, demonstrating that the source-destination pair can achieve significant benefits emanating from the competitive nature of the relays.
\end{abstract}

\section{INTRODUCTION}

Cooperative relaying paradigm in wireless communications relies on availability of relaying nodes that assist communications they do not benefit from [1]. As such, it is limited to scenarios involving either dedicated relays or altruistic nodes [2]. Recently, spectrum leasing for cooperation emerged as a new paradigm that extends the application range of cooperative communications to any network scenario, by motivating the relaying participation of 'regular' mobile terminals, that are typically non-altruistic nodes [3] , [4]. Thus, with this paradigm, the benefits of cooperative communications, such as increased network connectivity, decreased battery consumption, lower interference and reduced infrastructure requirements and cost, can be harvested in any network type.

Spectrum leasing prescribes that the nodes are rewarded for their relaying assistance through a fraction of source's bandwidth, and promises considerable gains for both the source and the relay [3]. Its potentials are recognized in variety of scenarios, e.g., for cooperative jamming-aided secure transmission [5], [6]. Among proposed spectrum leasing for cooperation schemes, [7] particularly stands out due to its flexibility in allocating the communication resources. In particular, it enables the source to employ a relay only to an extent that it finds beneficial, preserving the remaining bandwidth for its direct transmission, if necessary. Such a solution can be particularly useful for dynamic multihop scenarios. Moreover, unlike the previous mechanisms, it is based on interaction and negotiation between nodes explicitly about the leased bandwidth.

In this work, we extend the scheme proposed in [7] to include relay selection for a two-hop scenario with multiple relays. Such a scenario can frequently occur, as it can be expected that there are more than one terminal willing to gain additional spectrum access and improve their communication capabilities. The challenging part that needs to be addressed here is modeling the competitive nature of the involved nodes, and in particular that of the potential relays, and analyzing such system.

Similarly as in [7], the nodes' interaction is framed as Stackelberg game [8], with source acting as the game leader and the potential relays as a follower, contending over the amount of bandwidth dedicated for cooperation and relay's own data transmission. The new ingredient is that the competition between relays for bandwidth access is modeled using auctioning [9], [5] as a distributed resource allocation mechanism. Interaction between the source and the set of potential relays thus becomes a two-layer game, with Stackelberg game described above as the 'outer' framework, while the set of relays constitutes the follower entity whose cooperation level response is the auction outcome. Results demonstrate the benefits for the source node not only from availability of multiple cooperation alternatives, but also from the competitive nature of the relays. On the other hand, the competition between the relays decreases their performance comparing to a noncompetitive scenario.

\section{PRELIMINARIES}

In this section, we describe the system model and the utilities used in [7].

\section{A. System Model and Notation}

The model involves the source $\mathrm{S}$, destination $\mathrm{D}$, and nodes $\mathrm{S}_{i}, i=1, \ldots, N$, as illustrated in Figure 1 . The nodes are non-cooperative and self-interested [8]. The communication medium is the property of the node $\mathrm{S}$, that needs to communicate with $\mathrm{D}$. Nodes $\mathrm{S}_{i}$ also wants to transmit their data 


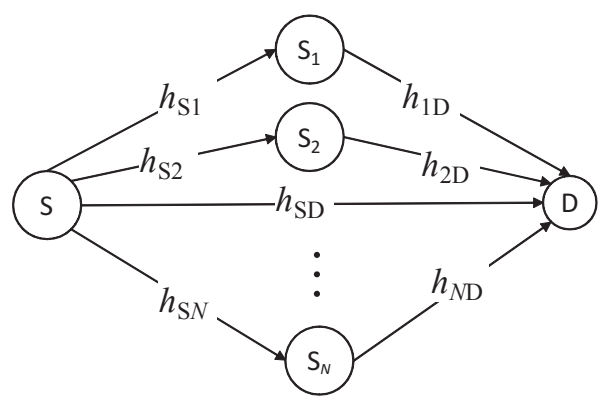

Figure 1. System model.

to $\mathrm{D}$, but avail no bandwidth. On the other hand, $\mathrm{S}$ would benefit from relaying services from $\mathrm{S}_{i}$, but the latter are unwilling to unconditionally assist the communication that excludes their data. Thus, the situation occurs where a trade between $\mathrm{S}$ and one of the nodes $\mathrm{S}_{i}$ can possibly benefit both. Namely, the node $S$ can employ one of the nodes $S_{i}$ as a relay, compensating it with a fraction of $S^{\prime}$ ' bandwidth/transmission slot for transmission of $\mathrm{S}_{i}$ 's own data.

Decode and forward is adopted as the relaying strategy. The approach can be extended to other schemes as well. Denote the transmission resource, e.g., the time slot, of node $\mathrm{S}$ as $T$ [sec]. Denote the time that $S_{i}$ will relay, expecting reward in return, as $\alpha_{i}<T$ [sec]. For generality, we also allow that $\mathrm{S}_{i}$ is willing to altruistically relay for the time $\alpha_{0, i}$. The spectrum reward from node $\mathrm{S}$ is a non-decreasing non-negative function of $\alpha_{0, i}$ and $\alpha_{i}, f\left(\alpha_{0, i}+\alpha_{i}\right)$ [sec]. As in [7], a linear function is used for simplicity, $f\left(\alpha_{0, i}+\alpha_{i}\right)=k\left(\alpha_{0, i}+\alpha_{i}\right)$, where $k$ is a parameter determined by $\mathrm{S}$. The model for interaction between the source and competitive potential relays to determine the parameters $\alpha_{i}$ and $k$ is elaborated in Section III.

The channels between nodes are constant during the interval of interest. The channel power gains for links between $\mathrm{S}$ and $\mathrm{D}, \mathrm{S}$ and $\mathrm{S}_{i}$, and $\mathrm{S}_{i}$ and $\mathrm{D}$, are denoted as $h_{S D}, h_{S i}$ and $h_{i D}$, respectively, $i=1, . ., N$. The transmission power of nodes $\mathrm{S}$ and $\mathrm{S}_{i}$ is denoted as $P_{S}$ and $P_{i}$, respectively, while the singlesided spectral density of the independent white Gaussian noise at receivers is $N_{0}$. Throughout the paper, we assume signaling using Gaussian codebooks and that the codebook is generated with average power $P_{S}$ or $P_{i}$ over the entire communication session. The generic formula for the link rates is thus given as

$$
R=\log _{2}\left(1+\frac{h P}{N_{0}}\right)
$$

The rates on links $\mathrm{S}-\mathrm{D}, \mathrm{S}-\mathrm{S}_{i}$, and $\mathrm{S}_{i}$-D are denoted as $R_{S D}, R_{S i}$ and $R_{i D}$, respectively, and calculated applying appropriate channel gain and power value in (1). Observe that by this formulation, the actual rate of a link is (1) scaled by the fraction the transmitter is on, leading to an average expended power scaled by the same fraction. The effect of this actual expended power shall be explicitly present in the utility of the relay via a pricing term. Finally, we note that a possible malicious nodes' behavior is out of the scope of this paper.

\begin{tabular}{|c|c|c|}
\hline$\left(\alpha_{0, i}+\alpha_{i}\right) / a_{i}$ & $k\left(\alpha_{0, i}+\alpha_{i}\right)$ & $T-\left(k+1 / a_{i}\right)\left(\alpha_{0, i}+\alpha_{i}\right)$ \\
S transmits and $\mathrm{S}_{i}$ relays & $\mathrm{S}_{i}$ transmits its data & S transmits using direct link \\
\hline
\end{tabular}

Figure 2. Slot allocation.

\section{B. Slot Allocation and Nodes' Utilities}

Assume that a relay $S_{i}$ is selected (relay selection process will be elaborated in Section III). Slot allocation resulting from the model described in Section II-A is illustrated in Figure 2. In particular, as shown in [7], the part of the slot allocated for the relaying is $\left(\alpha_{0}+\alpha\right) / a_{i}$, while the remaining $T-(k+$ $\left.1 / a_{i}\right)\left(\alpha_{0, i}+\alpha_{i}\right)$ is used for $\mathrm{S}_{i}$ 's direct transmission to $\mathrm{D}$, with $a_{i}=R_{S i} /\left(R_{S i}+R_{i D}\right)$.

As discussed in Section II-A, node $\mathrm{S}$ is looking to recruit $\mathrm{S}_{i}$ if it can increase its rate. In particular, $\mathrm{S}$ is willing to compensate the potential relay with a time $k\left(\alpha_{0, i}+\alpha_{i}\right)$ for relay's own data transmission while preserving the remaining time for its direct and cooperative transmission. Utility of the node $\mathrm{S}$ is the number of bits it can deliver to $\mathrm{D}$ :

$$
\begin{aligned}
U\left(k, \alpha_{i}\right) & =\left(\alpha_{0, i}+\alpha_{i}\right) R_{i D}+ \\
& \left(T-\left(k+\frac{1}{a_{i}}\right)\left(\alpha_{0, i}+\alpha_{i}\right)\right) R_{S D} .
\end{aligned}
$$

Conditions for the cooperation to be profitable for $\mathrm{S}$ are given in [7].

Unlike the node $S$ that 'starts' with non-zero number of delivered bits, $T R_{S D}$, the node $\mathrm{S}_{i}$ can not transmit any bits unless involved in relaying/reward scheme. To model such a node, one needs to carefully design its utility so as to prevent it from being taken advantage of, e.g., from being unrewarded, while preserving its goal of transmitting bits. Utility design for the relaying node is chosen as the difference between the satisfaction from accessing the spectrum and the cost for achieving such an access. In particular, utility is defined as

$$
W_{i}\left(\alpha_{i} ; k\right)=\log \left(1+k\left(\alpha_{0, i}+\alpha_{i}\right) R_{i D}\right)-c_{i} \alpha_{i},
$$

where the constant $c[1 / \mathrm{sec}]$ is the penalizing term given by the system and represents the (un)willingness of node to relay. Notice that the large/small value of $c$ would characterize a node that requires a significant/modest spectrum reward.

\section{Competitive Relay Selection}

This section addresses the relay selection problem for the proposed two-hop scheme involving multiple competitive potential relays. It is noted here that a relay selection method was also proposed in [7]. Although legitimate, the approach therein fails to fully exploit selfish and rational features of potential relays, which are likely to lead to contention for access to the bandwidth and consequently further improvement for the source [8]. In the following, we elaborate on the scheme that incorporates the competitive relays' behavior. 

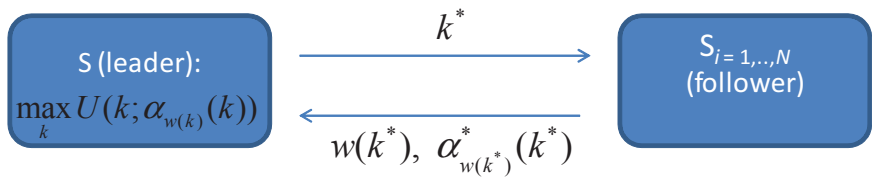

Figure 3. Interaction between $\mathrm{S}$ and $\mathrm{S}_{i=1, \ldots, N}$.

\section{A. Game-Theoretic Model}

To mimic a non-altruistic behavior, the nodes are defined as selfish and rational [8], and game theory is used as appropriate framework to analyze interaction between such nodes. Specifically, a convenient setting here is the Stackelberg game [8], with one agent, termed follower, acting subject to the strategy chosen by the other agent, leader, which in turns seeks maximization of its own utility. Here, the game leader is $\mathrm{S}$, as it is the legitimate operator in the spectrum, while the follower is the entity comprised of $\mathrm{S}_{i=1, \ldots, N}$.

The node $\mathrm{S}$ informs the follower about parameter $k$ and the follower's response $\alpha_{w(k)}^{*}(k)$ is the allocation outcome of the auction game played among relays, with $w(k)=1, . ., N$ indicating the winning relay, if any, for a source's strategy $k$, as detailed later. Interaction between the source and the set of potential relays thus becomes a two-layer game, with a Stackelberg game as the 'outer' framework, while the set of relays constitutes the follower entity whose cooperation level response is the auction outcome. This interaction is illustrated in Figure 3. The source's optimal strategy $k^{*}$ and the corresponding winner $w\left(k^{*}\right)$ and the allocation $\alpha_{w\left(k^{*}\right)}^{*}\left(k^{*}\right)$ are jointly referred to as the Stackelberg equilibrium.

The competition model is built upon the rules of Vickrey auction, i.e., the sealed-bid second-price auction [10] [9]. This auction type prescribes that the winning bidder is awarded with the bidding item at the price of the second largest bid, and is of particular interest due to its 'truthful bidding' property [10] [9]. Namely, bidders are motivated to bid with the maximum amount they would be willing to pay for the object. Importantly, such a strategic choice corresponds to the game-theoretic concept of dominant strategy equilibrium $(D S E)$, defined as the state wherein the strategies are required to remain preferable to every player irrespective of the amount of information available on the other players [8]. This property implies that the bidders require no information on other bidders' strategies or their evaluations of the bidding item. It is also noted that the considered framework is, however, not limited to Vickrey auctions and can be implemented via other auction types.

\section{B. Modified Vickrey Auction for Communication Model}

Here, the source acts as the auctioneer, the relays are bidders and the bidding article is the bandwidth, i.e., some fraction of the available slot. The auctioneer's utility $U\left(k, \alpha_{i}\right)$, when employing the relay $\mathrm{S}_{i}$, is defined in (2). The bidder $\mathrm{S}_{i}$ 's strategy is the relaying time fraction $\alpha_{i}$ and its utility $W_{i}$ is defined in (3), given that it is granted the spectrum access, i.e., that it won the auction, and zero otherwise.
For a given $k$, the bids are in the form of the source's utility $U\left(k, \alpha_{i}\right)$, implying that a bidder $\mathrm{S}_{i}$ must be also aware of the source's parameter $h_{\mathrm{SD}}$. The source sets the lowest acceptable rate, i.e., the reserve price, as its utility with no cooperation $U_{0}=T R_{S D}$, accepting only larger bids. Denoting the bidding strategies in the DSE equilibrium as $\alpha_{i}^{\text {bid }}$, the index of the winning relay for a given $k$ is

$$
w(k)=\arg \max _{i=1, . ., N} U\left(k, \alpha_{i}^{\mathrm{bid}}(k)\right),
$$

if $U\left(k, \alpha_{w(k)}^{\mathrm{bid}}\right)>U_{0}$, otherwise no relay is chosen. The standard assumption, also adopted here, is that, in the case of multiple equal highest offers, the situation is resolved by random allotment to one of them. Furthermore, the secondbest offer $U_{2}$ reads:

$$
U_{2}(k)=\max \left(\max _{i \neq w(k)} U\left(k, \alpha_{i}^{\mathrm{bid}}(k)\right), U_{0}\right) .
$$

Before proceeding to Section III-C to determine the DSE and the Stackelberg equilibria, we notice that the auctioning analytical tools are developed for simple linear or monotonic utility functions [9], which is not the case here. For a given $k$ satisfying the relay participation conditions in [7], the auctioneer's utility $U\left(k, \alpha_{i}\right)$ given in (2) is monotonically increasing in $\alpha_{i}$. However, for the same $k$, the bidding relay's utility $W_{i}\left(\alpha_{i} ; k\right)$ given in (3) is also monotonically increasing for $0 \leq \alpha_{i} \leq \alpha_{i, \max }(k)$, while it decreases for $\alpha_{i}>\alpha_{i, \max }(k)$, where $\alpha_{i, \max }(k)$ is the maximizer of $W_{i}\left(\alpha_{i} ; k\right)$ :

$$
\alpha_{i, \max }(k)=\left[\frac{1}{c}-\frac{1}{k R_{i D}}-\alpha_{0, i}\right]^{+},
$$

where $[x]^{+}=\max (x, 0)$. Such a shape of $W_{i}\left(\alpha_{i} ; k\right)$ indicates that it is possible to improve the DSE equilibrium for the communication model at hand and provides a guideline for finding the auction bids $\alpha_{i}^{\text {bid }}(k)$ and the auction outcome, $w(k)$ and $\alpha_{w(k)}^{*}(k)$ in Section III-C. Namely, unlike the setting in a baseline auction model, wherein the auctioneer and a bidder's utility are monotonically increasing and decreasing in price, respectively, i.e., a profit for one is a negative surplus to another [9], here the players' utilities have slopes of an equal signum and, thus, compatible goals for $0 \leq \alpha_{i} \leq$ $\alpha_{i, \max }(k)$. This observation leads us to propose the following modification of the Vickrey auction rule that can lead to the performance improvement for all the involved nodes in the communication model at hand.

Definition 1. In the proposed modification of Vickrey auction, the winning bidder is required to provide the source's utility that is at least the second largest bid, i.e., $U\left(k, \alpha_{w(k)}^{*}(k)\right) \geq$ $U_{2}(k)$.

The second largest bid $U_{2}(k)$ is given in (5). Unlike the original Vickrey principle, wherein the winning bidder provides the auctioneer with exactly the second-best price, this modification enables the winning node to choose a larger value if, as a result, its utility will increase. Notice that neither of the involved nodes, i.e., auctioneer and a bidder, are harmed by this deviation from the Vickrey principles, quite the opposite. 
The benefits due to this modification will be clearly visible in the following subsection.

\section{Equilibria}

The strategy for a bidder in the DSE is given by the following theorem.

Theorem 1. For a given $k$, the dominant bidding strategy $\alpha_{i}^{\text {bid }}(k)$ for a bidder $S_{i}$ is:

$$
\alpha_{i}^{\text {bid }}(k)=\min \left(\alpha_{i}^{\prime}(k), \alpha_{i}^{l i m}(k)\right) .
$$

In (6), the parameter $\alpha_{i}^{\prime}(k)$ is the solution of $W_{i}\left(\alpha_{i}>0 ; k\right)=$ 0 and reads

$$
\begin{aligned}
\alpha_{i}^{\prime}(k) & =-\frac{1+k \alpha_{0, i} R_{i D}}{k R_{i D}}- \\
& \frac{1}{c_{i}} \mathcal{W}_{-1}\left(-\frac{c_{i}}{k R_{i D}} e^{-\left(1+k \alpha_{0, i} R_{i D}\right) c_{i} / k R_{i D}}\right),
\end{aligned}
$$

where $\mathcal{W}_{l}(x)$ is the lth branch of of the multi-valued Lambert $W$ function. The parameter $\alpha_{i}^{\text {lim }}$ ensures that the slot duration $T$ is not exceeded and reads

$$
\alpha_{i}^{l i m}(k)=\frac{T}{k+\frac{1}{a_{i}}}-\alpha_{0, i}
$$

Proof: The proof is also illustrated in Figure 4, where two shapes of utilities $W$ are given. Since in the case of winning the auction, the bidder has no influence on the secondbest bid, the dominant strategy is to maximize the chance of winning, i.e., bidding with $\alpha$ as large possible, while ensuring that the outcome if winning the auction would remain in a feasible region of $\alpha$, i.e., that the slot duration is not exceeded, and that the resulting bidder's utility is no worse than if not participating, i.e., that it is non-negative. Thus, for $\alpha_{i}^{l i m}(k) \leq \alpha_{i}^{\prime}(k)$, which is the case (1) in Figure 4, optimal bid is $\alpha_{i}^{l i m}(k)$, as bidding with the larger value increases the chance for winning the auction only if resulting in an infeasible range, i.e., only if exceeding the slot duration. On the other side, for $\alpha_{i}^{\prime}(k)<\alpha_{i}^{l i m}(k)$, which is the case (2) in Figure 4, optimal bid is $\alpha_{i}^{\prime}(k)$, as bidding with the larger value increases the chances for winning the auction only if incurring a negative bidder's utility. Bidding with $\alpha$ smaller than $\min \left(\alpha_{i}^{\prime}(k), \alpha_{i}^{l i m}(k)\right)$ would only decrease the chance of winning. Thus, the optimal bidding value is given in (6).

The theorem is valid independent of whether Definition 1 is applied or not. Having won the auction, the winning relay $\mathrm{S}_{w(k)}$ has to provide the relaying time that produces at least $U_{2}(k)$. The following theorem provides this auction outcome.

Theorem 2. Under the rule in Definition 1, the strategy $\alpha_{w(k)}^{*}(k)$ chosen by the winning relays $S_{w(k)}$ is given by

$$
\alpha_{w(k)}^{*}(k)=\max \left(\alpha_{w(k)}^{m}(k), \alpha_{w(k), \max }(k)\right),
$$

where

$$
\alpha_{w(k)}^{m}(k)=\frac{U_{2}(k)-T R_{S D}}{R_{w(k) D}-\left(k+1 / a_{w(k)}\right) R_{S D}}-\alpha_{0, w(k)}
$$

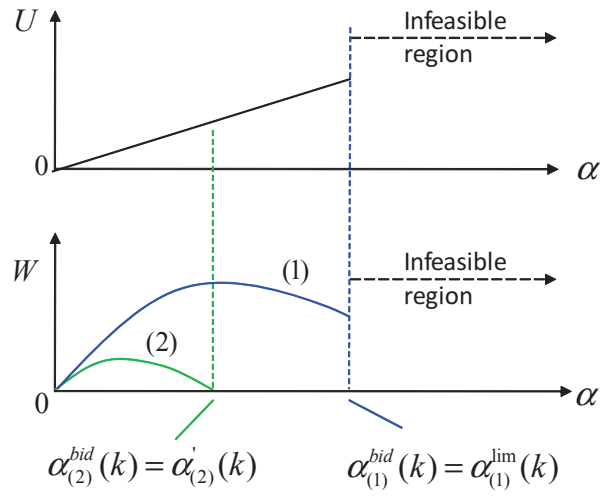

Figure 4. Illustration of Theorem 1 .

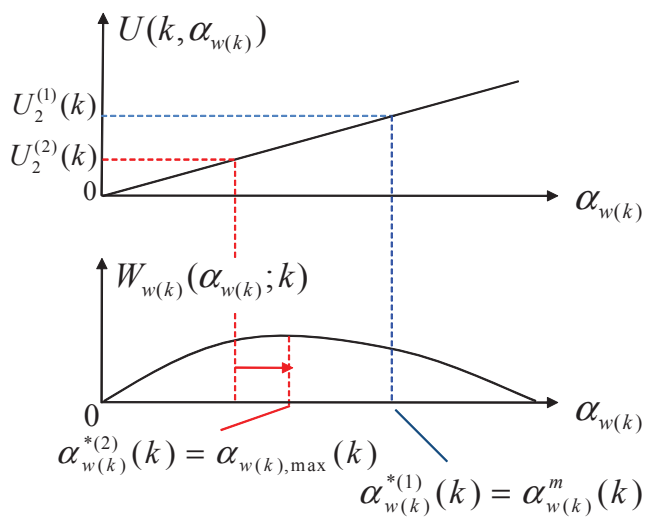

Figure 5. Illustration of Theorem 2.

and $\alpha_{w(k), \max }$ is given by (7). In particular, $\alpha_{w(k)}^{m}$ is the minimum $\alpha_{w(k)}$ that the $w(k)$-th (winning) relay needs to provide, given the second best bid $U_{2}(k)$, while $\alpha_{w(k), \max }$ can improve equilibrium performance for both the source and the winning relay, provided that $\alpha_{w(k), \max }>\alpha_{w(k)}^{m}$.

Proof: It suffices to show that (8) is the solution of the winning relay's $\mathrm{S}_{w(k)}$ utility maximization problem over $\alpha_{w(k)}$ under the constraint $U\left(k, \alpha_{w(k)}\right) \geq U_{2}(k)$. This maximization problem is also illustrated in Figure 5. If $U\left(k, \alpha_{w(k), \max }\right) \leq$ $U_{2}(k)$, i.e., if $\alpha_{w(k), \max } \leq \alpha_{w(k)}^{m}$, which is the case (1) in Figure 5, the winner chooses $\alpha_{w(k)}^{m}$ that corresponds to providing exactly the second-best price $U_{2}(k)$. If, however, $U\left(k, \alpha_{w(k), \max }\right)>U_{2}(k)$, i.e., if $\alpha_{w(k), \max }>\alpha_{w(k)}^{m}$, which corresponds to the case (2) in Figure 5, the winning bidder can exploit modification in Definition 1 and chooses $\alpha_{w(k), \max }$ that maximizes its utility $W_{w(k)}\left(k ; \alpha_{w(k)}(k)\right)$ and also increases the utility of the source.

Notice again in Theorem 2 and Figure 5 that for the case $\alpha_{w(k), \max } \geq \alpha_{w(k)}^{m}$, the alteration of Vickrey principle introduced by Definition 1 brings improvement to both the source's and relay's utility.

To conclude this section, the outcome of the auction, as given by (8), constitutes the follower's response in the Stack- 
elberg framework and the Stackelberg equilibrium thus reads

$$
k^{*}=\arg \max _{k} U\left(k, \alpha_{w(k)}^{*}(k)\right) .
$$

Notice that this model essentially requires that the source can anticipate the outcome of the auction game, and thus to be aware of all channel parameters in the system. An alternative to this requirement is for the source to perform a series of auctions with different $k$ and determine the most contributing one. As for the relaying nodes, a relay $S_{i}$ needs the knowledge of the parameters $h_{\mathrm{SD}}$, in addition to $h_{S i}$ and $h_{i D}$. On the positive side, a bidder requires no information on number of other bidders, their strategies or channel parameters, which is in line with the basic Vickrey implications.

\section{NumERICAL RESUlts}

In this section, we provide numerical results to illustrate performance of the proposed competitive relay selection scheme and to compare it with the scheme in [7]. As in [7], we assume flat Rayleigh fading on relay links, with $\mathbb{E}\left[h_{S i}\right]=\mathbb{E}\left[h_{i D}\right]=$ $\sqrt{2 / \pi} \cdot 2^{\gamma}, \mathbb{E}\left[h_{S D}\right]=\sqrt{2 / \pi}$, with $\gamma=4$. This illustrates scenario where the potential relays are placed in the middle of the line connecting $\mathrm{S}$ and $\mathrm{D}$, with the distance between $\mathrm{S}$ and $\mathrm{D}$ normalized to 1 , and $\gamma$ is the propagation factor. We further set $P_{S} / N_{0}=P_{1} / N_{0}=-10[\mathrm{~dB}], \alpha_{0, i=1, . ., N}=0$ and $T=1$ [sec]. The results describe the system performance in equilibrium.

In Figure 6 and Figure 7 we plot utilities of node $S$ and chosen relay node $S_{w\left(k^{*}\right)}$, respectively, averaged over channel realizations, that is $\mathbb{E}_{h}[U]$ and $\mathbb{E}_{h}\left[W_{w\left(k^{*}\right)}\right]$, versus the number of available relays $N$, for $c_{i}=c=0.1,1,5$ [1/sec]. Also shown in Figure 7 is the average number of transmitted bits for the chosen relay. Utilities are plotted for both the simple multirelay scheme in [7], denoted here as Stackelberg scheme, and the scheme proposed in this paper. The benefits of increased number of relays for the source can be clearly observed. Similar holds for the chosen relay, but one must recall that the utilities and rates of relays that are not chosen are zero. We also note that by taking competition into account, greater performance improvements are achieved for the source, while the performance of a chosen relay is decreased. This difference is more emphasized as the nature of the relays is more selfish, i.e., when the pricing factor $c$ increases. It is also worth commenting that for a scenario with the weaker relay channels $h_{S i}$ and $h_{i D}$, i.e., for a smaller $\gamma$ (not shown here), the relative performances for three depicted values of $c$ would differ. Namely, selfishness reflected in large parameter $c$ would be likely to harm the relay's performance instead of improving it, as was also discussed in [7].

\section{CONCLUding REMARKS}

In this paper, we have proposed a model for competitive relay selection in a flexible spectrum leasing scheme introduced in [7]. The game-theoretic framework models interactions between the nodes as a two-layer Stackelberg-auction game. Analytical and numerical results corroborate that competition for spectrum access between potential relays improve the performance of legitimate S-D pair.

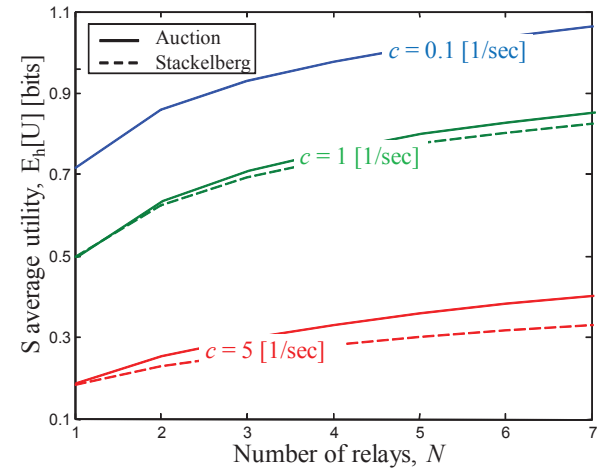

Figure 6. Average utility $\mathbb{E}_{h}[U]$ of node $\mathrm{S}$ versus number of relays $N$.

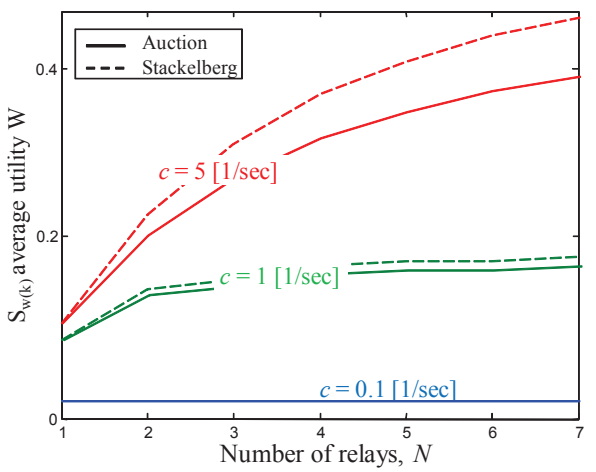

Figure 7. Average utility $\mathbb{E}_{h}[W]$ and average number of transmitted bits $\mathbb{E}_{h}\left[k^{*}\left(\alpha_{0, w\left(k^{*}\right)}+\alpha_{w(k)}^{*}\right) R_{w(k) D}\right]$ of a chosen relay versus number of relays $N$.

\section{REFERENCES}

[1] A. Sendonaris, E. Erkip and B. Aazhang, "User cooperation diversity Part I: System description," IEEE Trans. Commun., vol. 51, no. 11, pp. 1927-1938, Nov. 2003.

[2] L. Lai and H. El Gamal, "On cooperation in energy efficient wireless networks: the role of altruistic nodes," IEEE Trans. Wireless Commun., vol. 7, no. 5, pp. 1868-1878, May 2008.

[3] O. Simeone, I. Stanojev, S. Savazzi, Y. Bar-Ness, U. Spagnolini and R. Pickholtz, "Spectrum Leasing to Cooperating Secondary Ad Hoc Networks," IEEE Jour. Select. Areas Commun., vol. 26, no. 1, pp. 203213, Jan. 2008.

[4] I. Stanojev, O. Simeone, U. Spagnolini, Y. Bar-Ness and R. Pickholtz, "Cooperative ARQ via Auction-Based Spectrum Leasing," IEEE Trans. Commun., vol. 58, no. 6, pp. 1843-1856, June 2010.

[5] I. Stanojev and A. Yener, "Improving Secrecy Rate via Spectrum Leasing for Friendly Jamming," IEEE Trans. Wireless Commun., vol. 12, no. 1, pp. 134-145, Jan. 2013.

[6] I. Stanojev and A. Yener, "Recruiting Multi-Antenna Transmitters as Cooperative Jammers: An Auction-Theoretic Approach," Allerton Conference on Communication, Control and Computing, Allerton, Sep. 2011.

[7] I. Stanojev and A. Yener, "Facilitating Flexible Multihop Communication via Spectrum Leasing", in Proc. IEEE Intl. Symposium on Personal, Indoor and Mobile Radio Communications, PIMRC, Sydney, Australia, Sep. 2012.

[8] M. J. Osborne and A. Rubenstein, A Course in Game Theory, MIT Press, 1994.

[9] P. Klemperer, "Auction Theory: A Guide to the Literature," J. Economics Surveys, vol. 13, no. 3, pp. 227-286, Jul. 1999.

[10] W. Vickrey, "Counterspeculations, Auctions, and Competitive Sealed Tenders," Journal of Finance, vol. 16, pp. 8-37, 1961. 